\title{
Deterioro Cognitivo Leve: del concepto a la planificación de ejercicio físico
}

Mild Cognitive Impairment: From the concept to the planning of physical exercise

Juan Rivera Vargas ${ }^{1} \square$

\section{Resumen:}

Filiación:

1. Terapeuta físico, MSc. Rehabilitacion Funcional el Adulto Mayor, Clínica Santa Paula, Universidad Santa Paula, Curridabat, Costa Rica.

Correspondencia: $\square$ Juan Rivera Vargas juanmiriva@hotmail.com
Forma de citar: Rivera Vargas J. Deterioro cognitivo leve: del concepto a la planificación de ejercicio físico. Rev Ter. 2018;12(1):15-24.

Financiamiento:

Autofinanciado

Conflictos de Interés:

El autor declara no tener conflictos de interés en la presente publicación
Introducción: El envejecimiento implica una vulnerabilidad en el estado cognitivo debido a modificaciones funcionales y estructurales del cerebro. El objetivo de esta revisión fue brindar herramientas en la estructura, forma y elaboración de una intervención fisioterapéutica adecuada al sujeto con Deterioro Cognitivo Leve (DCL) basada en ejercicio y otros aspectos relevantes

Metodología: Se recopiló material bibliográfico de fuentes científicas primarias mediante la búsqueda en SciELO, MEDLINE. Se consideraron aquellas publicaciones escritas en idioma español e ingles, publicados del 2010 al 2018, utilizando los siguientes descriptores: neurogénesis, ejercicio y disfunción cognitiva. Se hallaron 65 artículos, de los cuales, después de una exhaustiva revisión se consideraron 29. Los mismos con un nivel de evidencia según Sackett entre I y III.

Discusión de Resultados: El ejercicio físico multimodal ha sido objeto de estudio debido a sus beneficios a nivel cerebral, como la sinaptogénesis, el mejoramiento de la vascularidad cerebral, optimización en redes atencionales, y favorecer los rendimientos cognitivos. Estos beneficios son proporcionales al tiempo de ejercicio realizado y pueden derivar en la mejoría del DCL en pacientes de ambos sexos y de cualquier edad. Se plantea un modelo de sesiones de ejercicio físico, flexible y sencillo que puede utilizarse por un profesional en terapia física para el abordaje del sujeto con DCL.

Conclusión: El ejercicio prescrito y supervisado por un profesional en terapia física trae beneficios no solo en el individuo con DCL, sino también en toda su red de apoyo y en los servicios de salud.

Palabras Claves: Deterioro cognitivo leve, terapia física, ejercicio físico, neurogénesis (Fuente: DeCS-BIREME) 


\section{Abstract}

Introduction: Aging implies a vulnerability on the cognitive state due to functional and structural modifications of the brain. The objective of this review is to provide tools on how to structure, form and elaborate a physiotherapy intervention adequate for the person with Mild Cognitive Impairment ( $\mathrm{MCl}$ ) based on exercises and other relevant aspects.

Method: A comprehensive search from primary sources from SciELO and MEDLINE databases was undertaken to base this study. All literature considered were publications from the years 2010 to 2018, in English as in Spanish idioms. The terms used were the following: Neurogenesis, exercise and cognitive impairment. A total of 65 studies were identified through primary search from which after an exhaustive review, 29 studies were included. These studies were then analyzed for quality assessment according to Sackett's level of evidence and reported classified between levels I and III.

Results: multimodal physical exercise has been subject of matter due to the brain benefits it provides such as synaptogenesis, improving cerebral vascularity, optimizing attentional networks and promoting cognitive abilities. The time these exercises are performed is proportional to the benefits achieved, which could lead to an equally improvement for the patients at any age and both genders with $\mathrm{MCl}$. A model (An alternative model) of flexible and simple sessions of physical exercise is presented in order to be used by a physical therapist for a clinical approach of a subject with $\mathrm{MCl}$.

Conclusion: the exercises prescribed and supervised by a professional on physical therapy lead to benefits not only for the individual with $\mathrm{MCl}$, but for all the support network and healthcare systems.
Key Words: cognitive dysfunction, physical therapy specialty, exercise, neurogenesis (Source: MeSHNLM)

\section{Introducción}

La cognición en su definición más simple, es concebida como la capacidad del ser humano para aprender, resolver problemas y darle un uso adecuado a la información almacenada ${ }^{1}$. Es un rasgo esencial y una característica fundamental para el óptimo desempeño de un individuo en su quehacer habitual.

El paso de los años en una persona, y las modificaciones que ocurren en el organismo a consecuencia de ello, se define como envejecimiento y se representa claramente por modificaciones estructurales, anatómicas y funcionales en sus diferentes sistemas orgánicos. Después de los 70 años, aproximadamente el $16 \%$ de las personas tiene deterioro cognitivo leve (DCL) y el $14 \%$ experimentan demencia ${ }^{1,2}$.La edad no solo se destaca como el principal factor de riesgo de $\mathrm{DCL}$, sino también de síndromes demenciales ${ }^{3,4}$

El Deterioro cognitivo puede llegar a evolucionar en su gravedad, es una entidad ocasionada por las consecuencias deletéreas de la $\operatorname{edad}^{5}$ y de otros factores comórbidos como la hipertensión arterial ${ }^{6,7}$, diabetes mellitus $^{8}$, enfermedad vascular cerebral ${ }^{9,10}$, enfermedad pulmonar obstructiva crónica, hiperlipidemias, trastornos del sueño ${ }^{11}$, problemas cardiaco $^{12}$, tabaquismo ${ }^{13}$, entre otros factores que actúan sobre la función cerebral de una persona deteriorándola.

En la actualidad son reconocidos y utilizados los criterios de Petersen ${ }^{14}$ para referirse a los subtipos del deterioro cognitivo leve (DCL) . A su vez el DCL ha sido propuesto por diversos autores como una etapa preclínica o prodrómica de la demencia tipo Alzheimer ${ }^{15}$. Samper J, Et al ${ }^{16}$ clasifica los distintos 
subtipos clínicos de DCL en: Deterioro Cognitivo Leve amnésico (DCLa), Deterioro Cognitivo Leve múltiples dominios (DCLmd) y Deterioro Cognitivo Leve dominio único no amnésico (DCLduna).

El término deterioro cognitivo para una persona y todo el núcleo familiar que lo rodea es nefasto pues se considera el punto de partida para demencia, perdida de independencia funcional y cercanía de la muerte. A este respecto, se debe de contemplar al adulto mayor desde todas sus perspectivas y abordar todos los componentes que favorezcan el enlentecimiento del deterioro cognitivo, por lo que la educación a todos los involucrados es un eje sumamente importante.

Uno de los problemas del DCL en la persona adulta mayor es su repercusión directa con su desempeño funcional y sus rendimientos en actividades ordinaria lo que puede evidenciar un mayor deterioro global ${ }^{17}$. Mirelman et al ${ }^{18}$ y Huckans et $a^{19}$, destacan que las dificultades en la marcha son frecuentes en las personas con deterioro cognitivo y el enlentecimiento motor puede predecir el inicio de deterioro cognitivo. Además, los autores destacan en su estudio, aplicado a 347 adultos mayores sin demencia pero con $\mathrm{DCL}$, que la calidad del movimiento y la generación del paso se observa disminuida en este tipo de sujetos, existe un menor movimiento de tronco hacia delante durante las transiciones, menor rotación axial durante los giros y mayor irregularidad en el paso, así como cambios en los mecanismos de control postural tales como menor agudeza visual y la atención ${ }^{20}$. EI DCL afecta además los rendimientos de estructuras neurales como el hipocampo, siendo éste el encargado del aprendizaje espacial y de la memoria declarativa, lo que provoca dificultades en dichas funciones en el individuo ${ }^{21}$

Siendo el DCL una situación en aumento y dada su implicación en las patologías neurodegenerativas tales como Enfermedad de Alzheimer, demencia por cuerpos de Lewy, Enfermedad de Parkinson y Corea de Huntington ${ }^{22}$, un grupo de consenso de la Asociación Internacional de Geriatría y Gerontología (IAGG-GARN) fue convocado en marzo de 2015 en St Louis Missouri, donde se enumeraron una serie de recomendaciones para el manejo del DCL, destacando: dieta mediterránea, ejercicio físico, actividades intelectuales y educación, como pilares básicos en la prevención secundaria del DCL y prevención primaria de un síndrome demencial no reversible ${ }^{1,23}$.

El objetivo de esta revisión es brindar herramientas en la estructura, forma y elaboración de una intervención fisioterapéutica adecuada al sujeto con DCL basada en el ejercicio y otros aspectos relevantes.

\section{Materiales y Métodos}

Se realizó una revisión bibliográfica de artículos disponibles en texto completo en los motores de búsqueda PubMed, Scielo, Redalyc y Elsevier, publicados entre el año 2010 y 2018, en idioma inglés y español, utilizando como descriptores: disfunción cognitiva, ejercicio, terapia física y neurogénesis enfocándose únicamente en la población adulta mayor. Se excluyeron artículos que hablaran de DCL asociado a un diagnóstico de demencia u otro trastorno neurológico.

La figura 1 describe el flujo para la obtención, análisis y recopilación de artículos científicos. Al ser sometidos a los criterios de exclusión, la cantidad de artículos incluidos fueron $29(\mathrm{~N}=29)$.

Los artículos seleccionados se distribuyeron según su nivel de evidencia y son representados en la Cuadro 1. 


\section{Discusión de resultados}

Beneficios de Ejercicio Físico en el adulto mayor con $\underline{D C L}$

De acuerdo a la literatura revisada, el ejercicio físico promueve la neurogénesis a nivel de zonas neurogénicas del cerebro adulto, tales como el bulbo olfatorio y el giro dentado del Hipocampo. De acuerdo

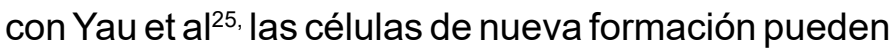
formar contacto sináptico con sus células diana en la tercera semana de maduración neural, y por si fuera poco, se ha determinado en roedores alrededor de 9000 nuevas células generadas en el hipocampo por día y que estos procesos serían similares en las regiones del cerebro humano.

Han sido extensamente estudiados los efectos de la actividad física y el ejercicio físico no solo durante la mediana edad, sino en el envejecimiento y su asociación con el menor riesgo de deterioro cognitivo o en el enlentecimiento del mismo ${ }^{26}$ ya sea por mecanismos primarios (acción del ejercicio en función cerebral, en factor neurotrófico derivado del cerebro (BDNF), en el factor de crecimiento insulínico 1 (IGF-1) y en el factor de crecimiento endotelial vascular (VEGF), entre otros) o por mecanismos

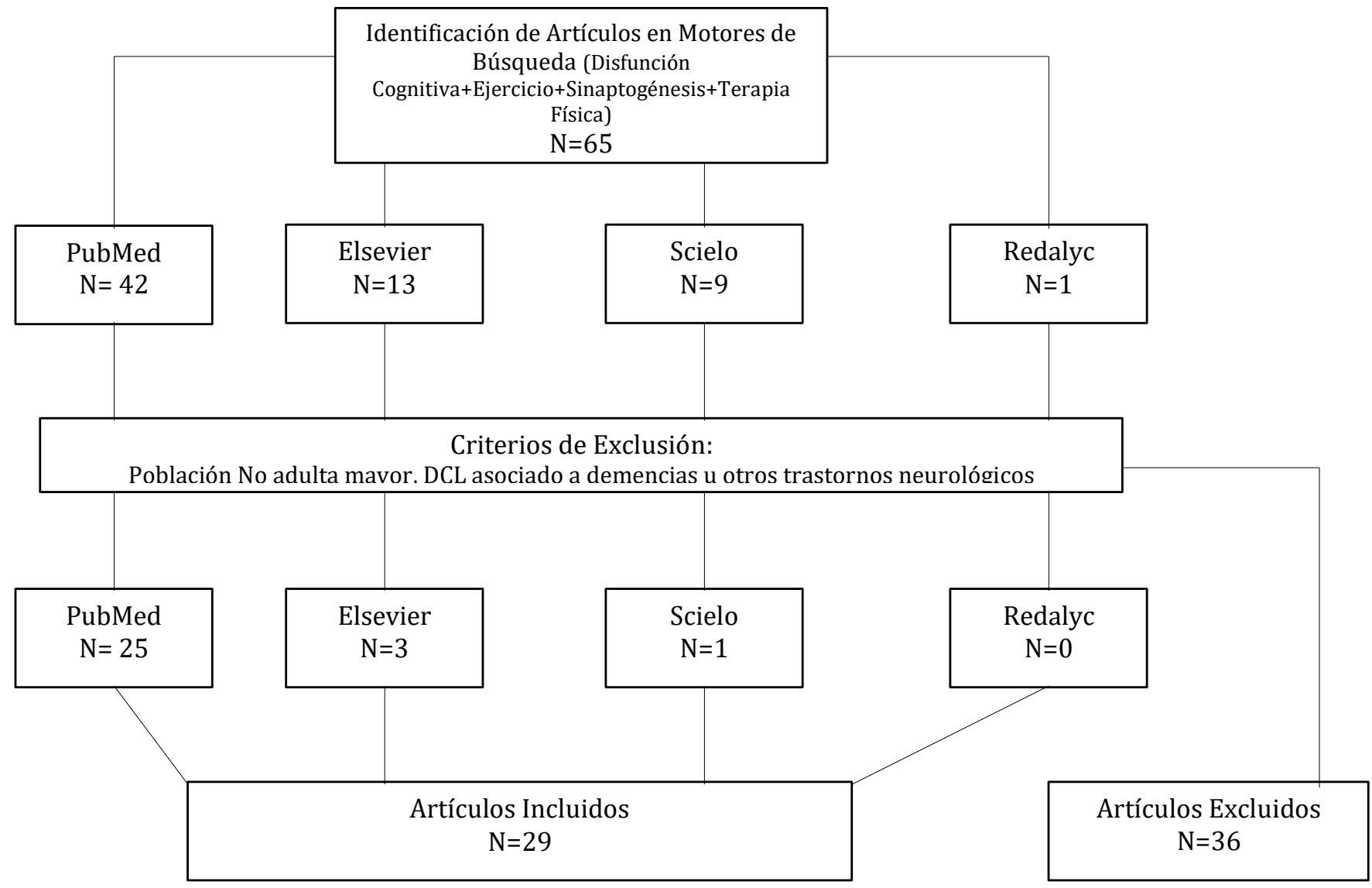

Figura 1. Diagrama de búsqueda bibliográfica.

Fuente: Elaboración propia 
secundarios mejorando la comorbilidad (regulando presión arterial, el perfil de lípidos en sangre, el nivel de obesidad, el sedentarismo, el sistema sanguíneo periférico y disminuyendo la ansiedad) del sujeto a través del ejercicio ${ }^{27-29}$.

Además de esto, se ha evidenciado que el ejercicio representa un regulador positivo para la neurogenesis del hipocampo, favorece la angiogénesis a partir de cambios en el volumen sanguíneo cerebral, contribuye en los mecanismos de plasticidad cerebral, aumenta la permeabilidad de la barrera hematoencefálica y normaliza los niveles de estrés oxidativo cerebral ${ }^{20}$.

\section{Intervención Fisioterapéutica basada en Ejercicio} para el DCL

Existe evidencia científica en la que se reconoce el uso de diversas formas de ejercicio físico para la persona con DCL, tales como el ejercicio aeróbico, el ejercicio de fuerza muscular y resistencia, e incluso componentes de equilibrio, coordinación y propiocepción, como elementos inherentes a la realización de ejercicio físico ${ }^{21,30,31}$. Así mismo, en los adultos mayores la practica regular de actividad física disminuye el riesgo de caídas y las complicaciones del sedentarismo ${ }^{32}$

Uno de los inconvenientes en la evidencia disponible en la actualidad, es que en múltiples investigaciones no se describe específicamente el tipo de ejercicio utilizado o la especificidad del mismo, sino, la combinación de ejercicios y esto no permite aclarar si la eficacia del ejercicio radica en la multi modalidad del ejercicio o es preferible enfocarse a un tipo específico del mismo, eso en esencia es uno de los objetivos primordiales del documento en cuestión, además claro está, de brindar al profesional en terapia física una adecuada dosificación y planificación de los planes de ejercicio físico.

\section{Cuadro 1. Distribución de artículos científicos según nivel de evidencia de Sackett ${ }^{24}$}

\begin{tabular}{|cc|}
\hline $\begin{array}{c}\text { Nivel de } \\
\text { Evidencia }\end{array}$ & $\begin{array}{c}\text { Cantidad de } \\
\text { Artículos }\end{array}$ \\
\hline I & 6 \\
II & 12 \\
III & 11 \\
IV & 0 \\
IV & 0 \\
Total & 29 \\
\hline
\end{tabular}

Es importante destacar que en los pacientes con DCL la causa originaria es multifactorial, lo que convierte al sujeto y a su intervención fisioterapéutica en única y heterogénea, con circunstancias propias y con personalidades que pueden abarcar desde apatía a las tareas propuestas e indiferencia hacia las mismas, hasta buena disposición y ánimo para las mismas. Por lo tanto, lo planteado a continuación es una forma de ejecución, más no, una imposición de normas o estilos, ya que se sabe que en todo proceso de rehabilitación es la intervención misma la que se adecua al sujeto y no al contrario como lamentablemente ocurre con alguna frecuencia.

La dosificación del ejercicio físico y toda su estructura de planificación en pacientes con DCL debe de ser realizada por profesionales en terapia física que tengan el manejo verbal y no verbal adecuado para este tipo de población, donde debe de cuidar aspectos tales como el tono de voz, la interacción con el sujeto (es mejor posicionarse en forma diagonal que de frente al paciente y una distancia promedio de 1 metro) y en la frecuente explicación para el inicio y demostración del acto motor, ya que errores en la misma, pueden propiciar eventos adversos, ante estos últimos, el profesional debe de conocerlos, reconocerlos y minimizarlos en la medida de lo posible ${ }^{33}$.

Como se expone en el Cuadro 2 toda sesión de 
ejercicio físico debe de iniciarse con un correcto saludo, indicando con propiedad el objetivo del día y el demostrarse interesado en el día del sujeto o en lo que éste quiera destacar durante el saludo. Además se recalca que la duración ideal debe de oscilar entre los 45 y 60 minutos por día esto con el fin de no llevar el sujeto a la fatiga, evitar eventos adversos y tomar en cuenta los requerimientos energéticos para lo que resta del día y al menos realizar el plan de ejercicio de 3 a 4 veces por semana idealmente. Se recomienda además, realizar los ejercicios físicos en horas de la mañana, donde hay mayores niveles de neurotransmisores excitatorios, mayor predisposición a la actividad física y mayor vigor ${ }^{21}$.

Posteriormente a esto, en reposo, se debe de tomar los signos vitales, específicamente la presión arterial, la frecuencia cardiaca y en casos específicos la glicemia y la saturación de Oxigeno. Se inicia la sesión, con 10 minutos de calentamiento, los cuales deben como mínimo de incluir ejercicios propioceptivos de la planta del pie, de la palma de la mano y de la zona dorso lumbar, seguido de entrenamiento de equilibrio estático y dinámico, balance y coordinación ojo-mano ${ }^{35-36}$.

Seguidamente se ejecutan labores psicomotrices con una duración de 10 minutos, en donde se llevan a cabo actividades motoras-espaciales como aceleramientos, desaceleramientos, giros, actividades que involucren sentarse-levantarse y canales de marcha, todo esto debe de realizarse en modalidad dual ${ }^{37}$, es decir, mientras el sujeto realiza la actividad se deben de ofrecer problemas cognitivos, como por ejemplo, mencionar en el minuto completo del canal de marcha todos los animales que recuerde o solo alimentos de color verde, etc.

Acto seguido, se ejecuta el ejercicio aeróbico, durante 15 minutos, el cual no es mas que la puesta en práctica de actividad en banda sin fin, ergometría de brazos, cicloergometro o remos, esto monitoreado mediante la escala de esfuerzo percibido (12-15 de esfuerzo percibido). En un estudio randomizado controlado que incluyó 100 adultos mayores con DCL, el ejercicio aeróbico resulto beneficioso para mejorar la memoria, mantener la función cognitiva y reducir la atrofia muscular presente en personas con DCL ${ }^{30}$.

Una práctica ideal, es que posterior a los ejercicios de tipo aeróbico se realicen ejercicios de fuerza muscular, pero en el paciente con deterioro cognitivo leve e incluso en cualquier sujeto con puntuaciones mayores a 10 en el Minimental State Examination (MMSE) se van a realizar de manera funcional, dividiéndose en 5 esferas de trabajo, las cuales son: a) balance estático y dinámico combinado con ejercicio de fuerza en miembros inferiores, b) balance dinámico mientras se camina, c) balance estático y dinámico cuando se está de pie, d) ejercicios de fuerza prensora y de miembros superiores $y$ e)estabilidad espinal. Estos son dosificados de acuerdo a la intensidad, catalogando a la misma en intensidades leves, medias y altas, siendo las primeras las que con menos peso $(\mathrm{kg})$ el paciente realiza el acto motor con repeticiones mayores a 15 , el de intensidades medias realizará repeticiones de 13 a 15 y el de intensidades altas los ejecutará de 8 a 12 repeticiones. Se recomienda utilizar en ejercicio con Pesas entre el $40-50 \%$ de su Resistencia Máxima $(\mathrm{RM})^{38-41}$.

Para finalizar la sesión de ejercicio físico, el enfriamiento debe realizarse durante 10 minutos (este tiempo debe ser el mismo que el asignado al calentamiento en adultos mayores) donde básicamente es llevar a la calma al sujeto por medio de ejercicios respiratorios, ejercicios de coordinación y repetición, tales como rebotar un balón en una superficie a un ritmo especifico marcado por el terapeuta o por la música de la predilección del paciente o seguir con partes de su cuerpo la melodía de canciones elegidas previamente a la sesión por 


\section{Cuadro 2. Intervención Fisioterapéutica de ejercicio físico en el Adulto Mayor con Deterioro Cognitivo Leve.}

\begin{tabular}{llll}
\hline $\begin{array}{l}\text { Tiempo de } \\
\text { Ejecución }\end{array}$ & $\begin{array}{l}\text { Fase del } \\
\text { Ejercicio }\end{array}$ & Tipos de Ejercicio & Consideraciones durante el ejercicio \\
\hline
\end{tabular}

10 minutos

Calentamien- 1.Respiratorios

to

2.Flexibilidad

3.Coordinación

4. Habilidades visoespaciales

15 minutos Aeróbico

De acuerdo a factores propios del paciente y recursos del TF.

Puede ser banda sinfín, caminadora, ergometría de brazos, ergometría de piernas

Calcular la Frecuencia Cardíaca al 70 al $80 \%$ de la Frecuencia
Fuerza

Muscular Cardiaca Máxima ${ }^{34}$

Utilizar escala de la conversación para monitorear durante el ejercicio(Talk Test) También se puede monitorear con oximetría de Pulso ${ }^{34}$

Se deben de trabajar los 6 ejes temáticos de alguna de dos maneras, ya sea funcional o tradicional con 8 a 12 repeticiones con un peso recomendado entre 40 y $50 \%$ de la Resistencia Muscular

Se fomenta la Interacción social. disociación ojo-mano) combinando al mismo tiempo con los 4 tipos de ejercicio. A esto se le conoce como componente dual.

\section{Escala de Esfuerzo Percibido \\ (debe de encontrarse entre12-15) ${ }^{34}$}

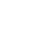

1. Fuerza en Miembros inferiores

2. Balance estático y dinámico en bípedo

3. Balance dinámico

4. Marcha (ya sea con cargas o peso, así como en distintas formas de terreno)

5. Prensión manual y Fuerza en miembros superiores

6. Estabilidad Espinal

Se deben de trabajar en estos 6 ejes temáticos: Enf

10 minutos
Se fomenta la Interacción social

Se deben de trabajar Labores psicomotrices (trabajo de memoria, concentración, orientación, trivias, adivinanzas, disociación ojo - mano) combinando al mismo tiempo con los 4 tipos de ejercicio.
4. Habilidades visoespaciales

2.Flexibilidad

3.Coordinación 
parte del paciente, entre otros. Esto genera una sensación de relajación, confort y se promueve la activación de redes atencionales y se estimula la reserva cognitiva².

La actividad física genera beneficios en múltiples sistemas del organismo humano, A nivel cerebral, algunos mecanismos neuroprotectores ocasionados por el ejercicio se exponen son el aumento del factor neurotrófico derivado del Cerebro (BDNF), aumento del factor de crecimiento insulínico 1 (IGF-1), aumento del factor de crecimiento endotelial vascular (VEGF), aumento en la facilitación de sinaptogénesis hipocampal, aumento de neurotransmisores excitatorios, aumento de testosterona y disminución de amiloide ${ }^{42-45}$.

Aunque no existe un protocolo de ejercicio físico en el paciente con deterioro cognitivo, lo anterior es una herramienta y un método flexible que permite al terapeuta físico llevar al máximo sus habilidades y aptitudes de entrenamiento, permitiendo la maleabilidad en las tareas y el margen de individualidad y por otra parte le permite al paciente ser estimulado por múltiples focos de intervención los cuales, no solo genera beneficios directos en su desempeño físico, sino a su vez en los desempeños funcionales, en el manejo del estrés, en la estructura del sueño y su percepción de salud ${ }^{37}$.

Así mismo el profesional que trabaje con pacientes portadores de DCL, debe ser un terapeuta físico especializado en rehabilitación funcional de la persona adulta mayor, ya que el paciente geriátrico representa uno de los grupos poblacionales más heterogéneos, por lo tanto, los cambios orgánicos propios del envejecimiento deben identificarse correctamente para diferenciarlos de las situaciones no asociadas al proceso normal de envejecimiento, de tal manera que las intervenciones contengan un ingrediente esencial: la integralidad del sujeto.

\section{Referencias Bibliográficas}

1. Morley JE, Morris JC, Berg Weger M, Borson $\mathrm{S}$, Carpenter BD, del Campo $\mathrm{N}$ et al. Brain health: The importance of recognizing cognitive impairment: An IAGG Consensus Conference. J Am Med Dir Assoc. 2015;16(9):731739.

2. Pineda DA. Envejecimiento, educación, hipertensión arterial, reserva cognitiva y deterioro cognitivo. Acta Neurol Colomb. 2016;32(2):89-90.

3. Fernandes Lopes RM, de Lima Argimon II. El entrenamiento cognitivo en los ancianos y efectos en las funciones ejecutivas. Acta Colomb Psicol. 2016;19(2):177-197.

4. Guix LM. Papel de los biomarcadores en el diagnóstico precoz de la enfermedad de Alzheimer. Rev Esp Geriatr Gerontol. 2011;46(Supl. 1):39-41

5. Moran RJ, Symmonds M, Dolan RJ, Friston KJ. The Brain Ages Optimally to Model Its Environment: Evidence from Sensory Learning over the Adult Lifespan. PLoS Comput Biol. 2014;10(1):e1003422

6. Intzandt B, Black SE, Lanctôt KL, Herrmann N, Oh P, Middleton LE. Is Cardiac Rehabilitation Exercise Feasible for People with Mild Cognitive Impairment? Can Geriatr J. 2015;18(2):65-72

7. Gottesman RF, Hillis AE. Predictors and assessment of cognitive dysfunction resulting from ischaemic stroke. Lancet Neurol. 2010;9(9):895-905.

8. Devenny KE, Sanders ML, Lawlor B, Olde Rikkert M, Schneider S. The effects of an extensive exercise programme on the pro- 
gression of Mild Cognitive Impairment (MCl): study protocol for a randomised controlled trial. BMC Geriatr. 2017;17:75.

9. Adell Serrano B, Perrot González JC, Escribano Stable DA, Castañeda Galeano VE, Usabiaga Bernal T, Aguilar Naranjo JJ. Relación entre reserva cognitiva y déficit cognitivo en el ictus. Rehabilitación. 2013;47(1):27-34.

10. Kirk Sanchez N, McGough E. Physical exercise and cognitive performance in the elderly: current perspectives. Clin Interv Aging. 2014;9:51-62

11. Langa KM, Levine DA. The Diagnosis and Management of Mild Cognitive Impairment: A Clinical Review. JAMA. 2014;312(23):25512561

12. Alosco ML, Spitznagel MB, Cohen R, Raz N, Sweet LH, Josephson R, et al. Decreased physical activity predicts cognitive dysfunction and reduced cerebral blood flow in heart failure. J Neurol Sci. 2014;339(1-2):169-175.

13. Henderson VW. Three midlife strategies to prevent cognitive impairment due to Alzheimer's disease. Climacteric. 2014;17(sup2):38-46.

14. López Álvarez J, Agüera Ortiz LF. Nuevos criterios diagnósticos de la demencia y la enfermedad de Alzheimer: una visión desde la psicogeriatría. Psicogeriatría. 2015;5(1):3-14

15. Albert MS, De Kosky ST, Dickson D, Dubois $\mathrm{B}$, Feldman $\mathrm{HH}$, Fox $\mathrm{NC}$, et al. The diagnosis of mild cognitive impairment due to Alzheimer's disease: Recommendations from the National Institute on Aging-Alzheimer's Association workgroups on diagnostic guidelines for Alzheimer's disease. Alzheimers Dement. 2011;7(3):270-279.
16. Noa J, Rodríguez J, Catases C, Pérez S. El deterioro cognitivo leve. Un paso antes de la enfermedad de Alzheimer. Rev Haban Cienc Med. 2011:10(1)27-36

17. Bostock S. A need for research and training involving patients with dementia. Int J Ther Rehabil. 2010;17(4):172-173.

18. Mirelman A, Weiss A, Buchman A. Association between performance on timed up and go subtasks and mild cognitive impairment: further insights into the links between cognitive and motor function. J Am Geriatr Soc. 2014;62(4):673-678

19. Huckans M, Hutson L, Twamley E, Jak A, Kaye J, Storzbach D. Efficacy of Cognitive Rehabilitation Therapies for Mild Cognitive Impairment $(\mathrm{MCl})$ in Older Adults: Working Toward a Theoretical Model and Evidence-Based Interventions. Neuropsychol Rev. 2013;23(1):63-80.

20. Marchetti M, Whitney S. Postural control in older adults with cognitive dysfunction. Phys Ther Rev. 2006;11(3):161-168.

21. Cejudo Jiménez J, Gómez Conesa A. Ejercicio físico en el tratamiento de la enfermedad de Alzheimer. Fisioterapia. 2011;33(3):111122.

22. Alves J. Non-pharmacological cognitive intervention for aging and dementia: Current perspectives. World J Clin Cases. 2013;1(8):233241.

23. Rodakowski J, Saghafi E, Butters MA, Skidmore ER. Non-pharmacological interventions for adults with mild cognitive impairment and early stage dementia: An updated scoping review. Mol Aspects Med. 2016;1(6)38-53. 
24. Manterola C, Asenjo Lobos C, Otzen T. Jerarquización de la evidencia. Niveles de evidencia y grados de recomendación uso actual. Rev Chilena Infectol. 2014;31 (6):705-718

25. Yau S, Gil Mohapel J, Christie B, So K. Physical exercise-induced adult neurogenesis: a good strategy to prevent cognitive decline in neurodegenerative diseases?. Biomed Res Int. 2014;403120.

26. Gates NJ, Sachdev PS, Fiatarone Singh MA, Valenzuela M. Cognitive and memory training in adults at risk of dementia: A Systematic Review. BMC Geriatr. 2011;11(1):55

27. Makizako $H$, Shimada $H$, Doi $T$, Park $H$, Yoshida D, Uemura K, et al. Poor balance and lower gray matter volume predict falls in older adults with mild cognitive impairment. BMC Neurol [Internet]. 2013 [citado 11 de abril de 2018];13(1). Disponible en: http://bmcneurol.biomedcentral.com/articles/10.1186/1471-2377-13-102

28. Sherrington C, Tiedemann A, Fairhall N, Close JCT, Lord SR. Exercise to prevent falls in older adults: an updated meta-analysis and best practice recommendations. New South Wales Public Health Bull. 2011;22(4):78-83

29. Zimerman M, Nitsch M, Giraux P, Gerloff C, Cohen LG, Hummel FC. Neuroenhancement of the aging brain: Restoring skill acquisition in old subjects. Ann Neurol. 2013;73(1):1015.

30. Suzuki T, Shimada H, Makizako H, Doi T, Yoshida D, Kato T, et al. A randomized controlled trial of multicomponent exercise in older adults with mild cognitive impairment. Plos One. 2013;8(4):e61483.

31. Gao J, Cheung RTF, Chan Y-S, Chu L-W,
Mak HKF, Lee TMC. The Relevance of ShortRange Fibers to Cognitive Efficiency and Brain Activation in Aging and Dementia. Hayasaka S, editor. PLoS One. 2014;9(4):e90307.

32. Ongel A, Hugener M. Impact of rejuvenators on aging properties of bitumen. Constr Build Mater. 2015;94:467-474

33. Margarit Jaso M, Gómez Conesa A. Programa de estimulación multisensorial para enfermos de Alzheimer: alteraciones de la conducta. Fisioterapia. 2008; 30(3):122-130.

34. Mijares GC, Carreto AM, Domínguez GSG. Eficacia del programa de rehabilitacion cardiaca en pacientes con cardiopatía isquémica. An Med (Mex). 2010;55(1):24-28

35. Blankevoort CG, van Heuvelen MJG, Scherder EJA. Reliability of Six Physical Performance Tests in Older People With Dementia. Phys Ther. 2013;93(1):69-78.

36. Venema DM, Bartels E, Siu K-C. Tasks Matter: A Cross-sectional Study of the Relationship of Cognition and Dual-Task Performance in Older Adults. J Geriatr Phys Ther. 2013;36(3):115-122.

37. Suzuki $M$, Kirimoto $H$, Inamura $A$, Yagi $M$, Omori Y, Yamada S. The relationship between knee extension strength and lower extremity functions in nursing home residents with dementia. Disabil Rehabil. 2012;34(3):202-209.

38. Kandola A, Hendrikse J, Lucassen PJ, Yücell $M$. Aerobic exercise as a tool to improve hippocampal plasticity and function in humans: practical implications for mental health treatment. Front Hum Neurosci. 2016;10:373

39. van Tilborg IA, Kessels RP, Hulstijn W. How should we teach everyday skills in demen- 
tia? A controlled study comparing implicit and explicit training methods. Clin Rehabil. 2011;25(7):638-648.

40. Vega JN, Newhouse PA. Mild Cognitive Impairment: Diagnosis, Longitudinal Course, and Emerging Treatments. Curr Psychiatry Rep [Internet]. 2014 [citado 11 de abril de 2018];16(10). Disponible en: http://link. springer.com/10.1007/s11920-014-0490-8

41. Voss MW, Prakash RS, Erickson KI, Basak C, Chaddock L, Kim JS, et al. Plasticity of brain networks in a randomized intervention trial of exercise training in older adults. Front Aging Neurosci. 2010;2(32):1-17.

42. Chapman SB, Aslan S, Spence JS, DeFina LF, Keebler MW, Didehbani N, et al. Shorter term aerobic exercise improves brain, cognition, and cardiovascular fitness in aging. Front Aging Neurosci. 2013;5(75):1-9.

43. Horne J. Exercise benefits for the aging brain depend on the accompanying cognitive load: insights from sleep electroencephalogram. Sleep Med. 2013;14(11):1208-1213.

44. Erickson KI, Voss MW, Prakash RS, Basak C, Szabo A, Chaddock L, et al. Exercise training increases size of hippocampus and improves memory. Proc Natl Acad Sci USA. 2011;108(7):3017-3022.

45. Brown BM, Peiffer JJ, Martins RN. Multiple effects of physical activity on molecular and cognitive signs of brain aging: can exercise slow neurodegeneration and delay Alzheimer's disease? Mol Psychiatry. 2013;18(8):864-874. 